\title{
Merging the ultrasonic and microacoustic sensor principles
}

\author{
Ralf Lucklum $^{1 *}$, Manzhu Ke ${ }^{1,2}$, Mikhail Zubtsov ${ }^{1}$ \\ ${ }^{1}$ Institute of Micro and Sensor Systems (IMOS), Otto-von-Guericke-University Magdeburg, Germany \\ ${ }^{2}$ Department of Physics, Wuhan University, China
}

\begin{abstract}
Ultrasonic sensors and acoustic (resonant) microsensors are well accepted devices in many application fields. Both principles are based on acoustic wave propagation where the value of interest perturbs wave propagation in a distinct manner. Acoustic band gap materials, so-called phononic crystals, provide a novel and alternative platform for sensing material properties in small cavities. The sensor employs specific transmission windows within the band gap to determine properties of a component that builds the phononic crystal. The value of practical interest changes its acoustic properties of the material, thereby changing the acoustic properties of the phononic crystal. Transmission or reflection coefficients are appropriate parameters for measurement and used to localize a transmission peak within the band gap of the phononic crystal. The frequency of maximum transmission acts as measure. The capability of the concept will be demonstrated with a one-dimensional arrangement of solid plates and liquid filled cavities and a two-dimensional periodic arrangement of liquid filled holes in a solid matrix.
\end{abstract}

Keywords: Phononic crystal sensor; Ultrasonic sensor; Microacoustic sensor, Resonant Sensor

\section{Introduction and motivation}

Acoustic waves at ultrasonic frequencies are used in a large variety of ways. One example is their use for sensing. Besides non-destructive testing and level or flow meters, an attractive application of ultrasonic sensors has been established in chemical process monitoring and control. Ultrasonic sensors utilize transducers which transform an electrical signal into an acoustic wave and vice versa. The general scheme of this kind of sensors comprises an acoustic wave penetrating into and traveling through the medium of interest thereby probing its acoustic properties. These properties include intrinsic material parameters (density, viscosity, elastic moduli, speed of sound, ...) and geometric values (layer thickness, acoustic path length, ...). For example, the time of flight of an ultrasonic impulse or short burst is determined jointly by speed of sound of the medium and distance between transmitter and receiver. If one value is known, the other one can be determined. Ultrasonic sensors in process industry for example, work with a well-defined distance between transmitter and receiver and therefore determine the speed of sound of the medium between the transducers with high accuracy [1]. Determination of material properties therefore relies on their correlation to speed of sound.

Acoustic microsensors belong to the group of resonant sensors. Their basic principle of operation is a traveling ultrasonic wave in a confined structure that produces a standing wave. Its frequency is determined jointly by the velocity of the traveling wave and the dimensions of the confinement structure. To keep the Q-factor of the resonator high, a mode is utilized which only evanescently penetrates the surrounding medium. The common background of acoustic microsensors is hence the sensitivity of the resonance frequency to changes of the acoustic properties at the interface between the resonator and the surrounding medium.

The resonance frequency can be measured with very high accuracy as well. It has been shown that the acoustic impedance acting at the device surface is the key parameter in the sensing process. This effective impedance may change due to a simple mass change of a chemically sensitive layer immobilized on the resonator surface as used for the so-called mass balance, or may be caused by viscoelastic properties of the coating material or viscosity changes of a semi-infinite liquid facing the resonator [2].

The task of ultrasonic and acoustic sensors is not merely to detect ultrasound or to determine time-of-flight or resonance frequency, respectively. They must provide the information carried by the ultrasonic wave. Furthermore, this information must have a valuable correlation to the parameter of interest. This can be a direct value like liquid level in a tank or density or viscosity of

$\bar{*}$ corresponding author, email: ralf.lucklum@ovgu.de 
a liquid. In many other cases indirect values have higher priority, like conversion rate in a chemical reactor or concentration of a toxic gas in air.

In summary, ultrasonic sensors and acoustic microsensors have some features in common and some distinguishing from each other. Both are based on acoustic waves at ultrasonic frequencies. The ultrasonic wave carries the information of interest. For ultrasonic sensors a short ultrasonic burst is generated and received from transducers usually isolated from the medium. The ultrasonic wave is perturbed while traveling through the medium. The primary measure is the time-of-flight. Acoustic microsensors apply the continuous wave mode and the primary measure is the resonance frequency of the acoustic resonator. The acoustic wave travels in the acoustic microsensor. Its propagation is perturbed by changes in the boundary conditions caused by the medium, which is in direct contact to the resonator (Fig. 1a and b).

Nowadays microfluidic devices appreciate increasing interest in research and development. The number of sensors available for those systems is still rather limited. This especially applies to sensors measuring material properties in microchannels or microreactors. At microscale ultrasonic sensors would face a severe limitation in resolution in time-of-flight measurements with a decreasing distance between transmitter and receiver. Taking a typical microreactor having a width of around $1 \mathrm{~mm}$ and sound velocities of liquids between $v \approx 900 . .1600 \mathrm{~ms}^{-1}$, the wavelength in liquids is in the same order of magnitude when working with common ultrasonic transducers having resonance frequencies of a few MHz. The time of flight is about or less than $1 \mu$ s. Since in technical processes the minimum resolution of speed of sound is $0.1 \%$ to achieve a relevant resolution of the value of interest a resolution in time of flight of less than $1 \mathrm{~ns}$ is required. This value is not a general problem; however, the effort which must be spent on transducers and electronics would be much higher. On the other hand, acoustic microsensors would require the integration of high-Q resonators into the microfluidic system which is also a challenging task. Piezoelectric resonators are not compatible with standard MEMS technology whereas many micromachined resonators like cantilevers do not facilitate pure in-plane displacement and hence provide only low Q-factors. MEMS compatible sensor technologies under development are based on deposited piezoelectric thin films as used for FBARs [3], furthermore based on CMUTs [4,5] or on magnetic direct excitation [6].

Here we present a novel concept of material property determination of liquids confined in small cavities. It is based on phononic crystals. Phononic crystals are periodic composite materials with periodic modulation of elasticity, mass density and longitudinal and transverse velocities of elastic waves. The typical structure consists of scattering centers with elastic properties different to a homogeneous matrix surrounding the scatters (Fig. 1c) [7]. Phononic crystals are somehow similar to photonic crystals [8] and can be understood as the acoustic analogue.

A phononic crystal is essentially made of homogeneous spherical or cylindrical inclusions arranged periodically in a homogeneous host medium. Typically cylinders are arranged as in a square, hexagonal or honey-comb lattice. The dimension involved in phononic crystals, i.e. the lattice parameter, range from a few meters down to a few micrometers or hundreds of nanometers.

The most important feature of phononic crystals are frequency regions, termed complete or absolute band gaps, within which (ultra)sound cannot propagate through the structure, independent of polarization and angle of direction of wave propagation. The focus of actual studies has moved to wave guiding, bending or wave confinement as a result of point or linear defects [9-12], or the interaction of acoustic with other kinds of waves, especially the photon-phonon interaction in

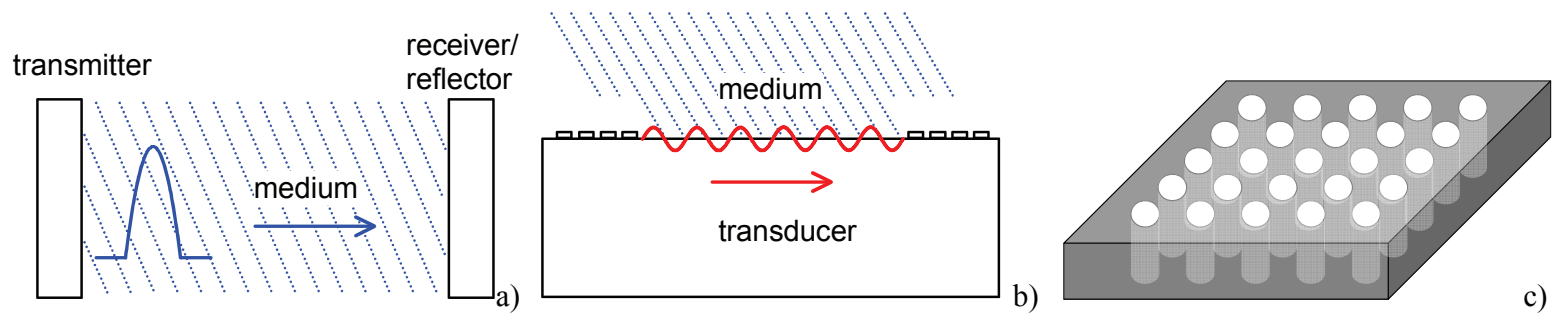

Fig. 1: Scheme of ultrasonic sensors, acoustic microsensors and phononic crystal with solid matrix and cylindrical holes 
periodic structures [13]. Although most research deals with the rich physics of phononic crystals, first applications arise in acoustic shielding, mirrors, filters and demultiplexers. The application as sensor is just emerging. As always, the sensor concept is based on a correlation of a characteristic feature of the phononic crystal and the value to be measured. The concept we have introduced in $[14,15]$ exploits the frequency of a transmission window within the band gap. We could show theoretically and experimentally that this frequency shifts with speed of sound of a liquid confined in the solid matrix.

For the band gap appearing at frequencies at several $100 \mathrm{kHz}$ up to a few $\mathrm{MHz}$ range, the required periodicity varies in range of millimeters to $100 \mathrm{~s}$ of micrometers, exactly the dimension range of microfluidic cavities. Higher probing frequencies give access to smaller dimensions. The promising feature of phononic crystals when working with liquids confined in a regular structure is the existence of modes which cause very narrow transmission windows in the band gap, also called transmission peaks. Acoustic energy is localized in a small region which acts as point of measurement. It exhibits resonant characteristics and therefore provides a simple measure, providing the position of the respective mode on the frequency scale is related to cavity properties or the material therein. In this way the measurement of a 'resonance' frequency (frequency of maximum transmission) as applied for acoustic microsensors and wave propagation through the medium as applied for ultrasonic sensors is merged in a new sensor platform based on phononic crystals.

\section{Background}

The concept of band gaps can be explained by the multiple scattering of waves within a phononic crystal. When a set of scatters is positioned periodically in the matrix, waves are strongly scattered among the scatters. A band gap appears when having destructive interference of waves happens in a given direction.

Several numerical methods have been developed to investigate the band structures of elastic waves propagating in phononic crystals as well as the transmission through it. We have been applying the Layer Multiple Scattering Theory (LMST) [16] to finite two-dimensional phononic crystal slab with a certain number of layers of cylindrical scatters, as well as COMSOL Multiphysics and recently the Finite Integration Theory (FIT). The Transmission Line Model (TLM) has been used for transmission calculations through a one-dimensional approximation consisting of metal plates and liquids in-between. The TLM is much faster to compute, therefore general tendencies important for a sensor application have been studied with the TLM first. Those benchmarks are the sensitivity, peak half band width, shape, number and separation of transmission peaks. The sensitivity of the sensor, $S_{f}$ can be defined as the ratio of frequency shift, $\Delta f$, and change of any input parameter, $\Delta x$ :

$$
S_{f}=\frac{\Delta f}{\Delta x}
$$

The sensitivity has been found to be dependent on the probing frequency, $f_{0}$. Furthermore, in terms of the detection limit the peak half band width, $f_{H B W}$, must be considered, hence the reduced sensitivity, $S_{f r}$, gives much better insights to the sensor capabilities:

$$
S_{f r}=\frac{\Delta f}{\Delta x f_{0} \Delta f_{H B W}}
$$

\section{Results}

We demonstrate the validity of the phononic crystal sensor concept in two steps. We first report on theoretical and experimental results achieved with the reduced one-dimensional structure. Modeling has been performed on the basis of analytical solutions using a specific geometry and material properties from literature. Experiments have been performed with an arrangement of metal plates and liquid filled cavities sufficiently large in lateral dimensions to fulfill the approximations 
made. In the second step we describe results with a two-dimensional structure with holes periodically drilled into a solid matrix. We especially concentrate on transmission features similar to those of the 1D analysis when all holes are filled with the liquid of interest. We finally report on a specific arrangement characterized by an incidence direction of the acoustic wave perpendicular to the plate.

Fig. 2 shows the calculated reflection coefficient and the measured transmission through a 1D phononic crystal made of aluminum plates $\left(\rho_{A l}=2720 \mathrm{~kg} \mathrm{~m}^{-3}, v_{A l}=6170 \mathrm{~m} \mathrm{~s}^{-1}, h_{A l}=3 \mathrm{~mm}\right)$, inbetween $900 \mu \mathrm{m}$ thick layers of DI-water, propanol and mixtures thereof (for data see Table 1). The phononic crystal is completely immersed into a DI-water-filled large container. The piezoelectric transducers are placed about $10 \mathrm{~mm}$ away from the phononic crystal. Due to their finite size standing waves in the container appear between transducer and crystal. The regular four calculated peaks are in coincidence with those experimentally found, however, due to the transducer parameters $\left(f_{0}=1 \mathrm{MHz}, f_{H B W}=45 \mathrm{kHz}\right)$ the one at $642 \mathrm{kHz}$ almost vanishes. These peaks change their position with the distance between the transducer and the phononic crystal but

Table 1: Density and speed of sound of materials

\begin{tabular}{lrc}
\hline $\mathrm{x}_{2}$ & $\begin{array}{l}\text { density } \rho \\
\left(\mathrm{kgm}^{-3}\right)\end{array}$ & $\begin{array}{l}\text { speed of sound } c \\
\left(\mathrm{~ms}^{-1}\right)\end{array}$ \\
\hline $0 \quad$ (water) & 998 & 1483 \\
0.021 & 990 & 1545 \\
0.035 & 982 & 1578 \\
0.056 & 974 & 1588 \\
0.102 & 956 & 1531 \\
0.158 & 933 & 1472 \\
0.230 & 908 & 1421 \\
1 (1-propanol) & 804 & 1220 \\
1 (2-propanol) & 777 & 1126 \\
\hline
\end{tabular}

do not move when changing the liquid in the cavity between the metal plates. The frequency of the additional peak marked with an arrow has been found at $775 \mathrm{kHz}$ for water and $622 / 628 \mathrm{kHz}$ (model/experiment) for propanol. This deviation is less than that calculated from different literature data [1719]. This peak does not move with the distance between transducer and phononic crystal. We furthermore have tested mixtures of water and propanol and could recover the characteristic maximum at a molar ratio of 0.056 as a result of a maximum in speed of sound (Fig. 3, Table 1). For a concentration range between $x_{2}=0 \ldots 0.035$ of 2-propanol $(0 \ldots 10 \%)$ one can estimate a limit of detection of about $\Delta x_{2}=0.1 \%$ [20].

The theoretical analysis of 2D phononic crystals reveals the same features, especially a band gap and the appearance of transmission windows within the band gap when filling the holes with a liquid. However, the number of peaks is quite large and makes measurement rather complicated. Fig. 4 shows the transmission spectrum for empty and water filled holes for an optimized crystal having a lattice parameter of the square array of holes of $1.0 \mathrm{~mm}$ and the filling ratio of 0.6.

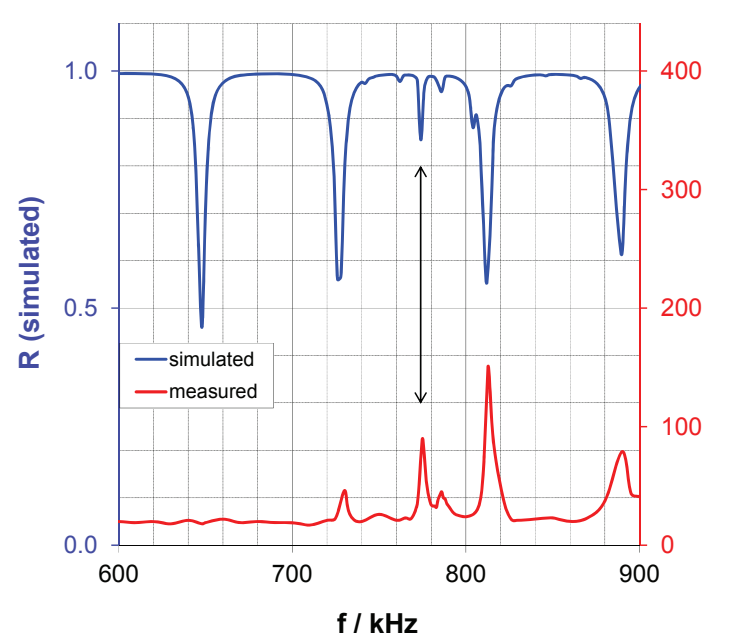

Fig. 2: Reflection coefficient magnitude (left axis, blue) calculated with a modified transmission line model and measured peak-to-peak voltage (right axis, red) for a single gap 1D single phononic crystal sensor [20].



Fig. 3: Dependence of frequency of maximum transmission through a phononic crystal sensor. The solid curve represents the frequency calculated from literature values, the dots represent experimental data [20]. 
The transmission spectrum of longitudinal waves depicts a band gap between $1.4 \mathrm{MHz}$ and $2.36 \mathrm{MHz}$ when the holes are empty (a). When filling water in all holes (b) the band gap moves to slightly lower frequencies between $1.26 \mathrm{MHz}$ and $2.07 \mathrm{MHz}$. The transmission peaks within the band gap move downwards with decreasing speed of sound, e.g. the first one about $260 \mathrm{kHz}$ when replacing water by propanol [20]. Optimization especially regarding separation of the transmission peak by reducing lattice symmetry has been reported in [21].

Besides the common in-plane incidence of ultrasonic waves we have analyzed the transmission behavior of longitudinal waves through a phononic crystal slab with normal incidence of ultrasonic waves as well. An extraordinary transmission appears as reported in [22]. The hole diameter is in the sub-wavelength range with respect to the frequency of maximum transmission. Again, the extraordinary transmission is supported by resonance phenomena in the phononic crystal structure. The peak frequency position is completely defined by material properties of the participating materials and geometry (hole radius and lattice constant) and changes with the composition of our water-propanol mixture. The half band width of this peak is larger and the reduced sensitivity (eq. 2) of the device is smaller than in the other realizations; however, the transmission peak is more robust under experimental conditions. In contrast to the experiment with the one-dimensional sensor, the phononic crystal plate is fully immersed into the liquid to be analyzed, i.e., the liquid mixture fills the holes and covers the surface of the plate. The transducers have been placed about $10 \mathrm{~cm}$ away to reduce the standing wave effect. To compare the frequency of the transmission peak with the theoretical prediction as well as the amplitude the peak-to-peak voltage has been 'normalized' with the peak-to-peak voltage measured under the same conditions without the hole array plate in the transmission path. Fig. 5 shows calculated and measured transmission spectra for three sample mixtures. Whereas the systematic error between theory and experiment has been found to be caused by deviations in the actual geometry of the fabricated device, the decreased amplitude of the extraordinary transmission peak with increasing propanol concentration is still under discussion.
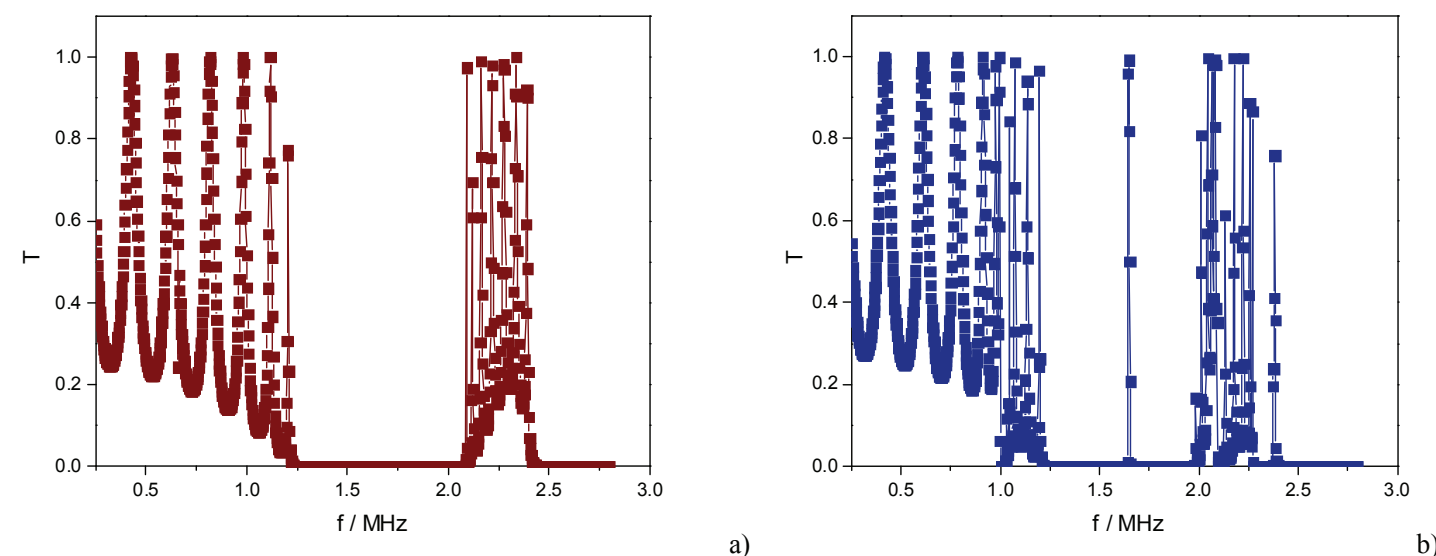

Fig. 4: Transmission spectrum of longitudinal waves through a 2D phononic crystal. The holes are empty (a) or filled with DI-water (b) [23].
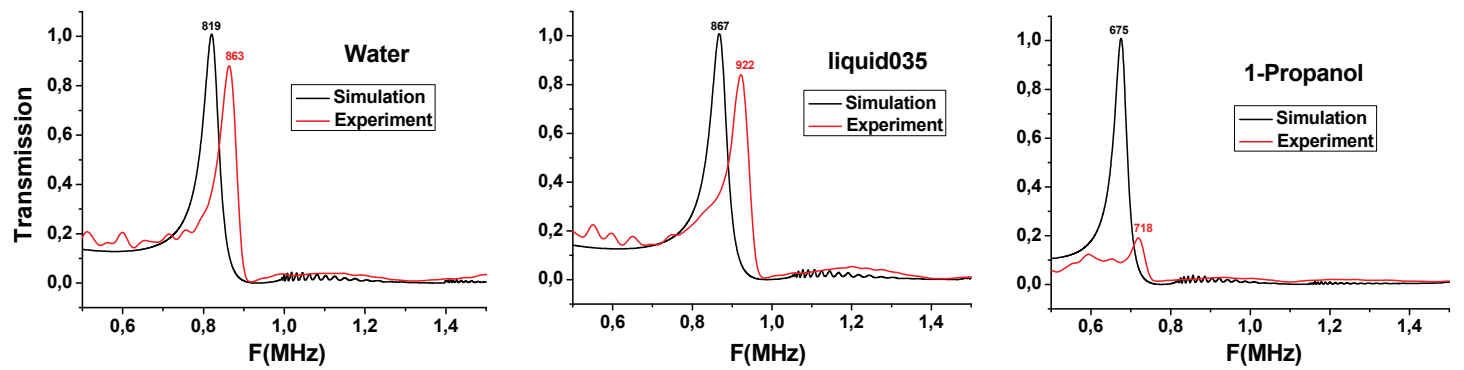

Fig. 4: Transmission spectrum of longitudinal waves through a 2D phononic crystal with incidence of sound at normal direction for water, a water-propanol mixture with $\mathrm{x}_{2}=0.35$ and pure 1-propanol. 


\section{Conclusions}

Phononic crystals provide a new platform for the determination of liquid properties in small cavities. Transmission windows which appear when the cavities are filled with a liquid display resonance features. The respective peak 'resonance' frequency is basically governed by the speed of sound of the liquid. The feasibility of a two-dimensional phononic crystal, where holes are drilled in a solid matrix act as liquid containers, could be demonstrated. A sensitivity comparable to ultrasonic sensors and acoustic microsensors could be achieved.

\section{Acknowledgements}

The work has been supported by a grant of the German Research Foundation (Lu 606/12-1) and the European Commission Seventh Framework Program (233883, TAILPHOX) which is gratefully acknowledged.

\section{References}

1 P. Hauptmann, N. Hoppe, A. Puettmer, "Application of ultrasonic sensors in the process industry", Meas. Sci. Technol. 13 (2002), pp. R73-R83.

2 R. Lucklum, C. Behling, P. Hauptmann, "Role of Mass Accumulation and Viscoelastic Film Properties for the Response of Acoustic-Wave-Based Chemical Sensors", Anal. Chem. 71 (1999), pp. 2488-2496.

3 J. Weber, W.M. Albers, J. Tuppurainen, M. Link, R. Gabl, W. Wersing, M. Schreiter, "Shear mode FBARs as highly sensitive liquid biosensors", Sens. Act. A 128 (2006), pp.84-88.

4 K. K. Park, H. J. Lee, G. G. Yaralioglu, Ö. Oralkan, M. Kupnik, C. F. Quate, B. T. Khuri-Yakub, T. Braun, H. P. Lang, M. Hegner, Ch. Gerber, J. Gimzewski, "Capacitive micromachined ultrasonic transducers for chemical detection in nitrogen", Appl. Phys. Lett. 91 (2007), pp. 094102:1-3.

5 Y. Li, R. Lucklum, P. Hauptmann, "2-Step Surface Modification Technology for Acoustic Chemical Sensor Arrays based on CMUTs", 2008 IEEE Int. Ultrason. Symp. Proc., pp. 1030-1033.

6 F. Lucklum, B. Jakoby, "Novel magnetic-acoustic resonator sensors for remote liquid phase measurement and mass detection", Sens. Act. A 145-146 (2008), pp. 44-51.

7 M.M. Sigalas, E.N. Economou, "Elastic and acoustic wave band structure", J. Sound Vibr. 158 (1992), pp. $377-382$.

8 J.-M. Lourtioz, H. Benisty, V. Berger, J.-M. Gerard, D. Maystre, A. Tchelnokov, "Photonic Crystals: Towards Nanoscale Photonic Devices", Springer, Berlin Heidelberg, 2008.

9 X. Li, Z. Liu, "Bending and branching of acoustic waves in two-dimensional phononic crystals with linear defects", Phys. Lett, A 338 (2005), pp. 413-419.

10 X. Li, Z. Liu, "Coupling of cavity modes and guiding modes in two-dimensional phononic crystals", Solid State Commun. 133 (2005) pp. 397-402.

11 A. Khelif, P.A. Deymier, B. Djafari-Rouhani, J.O. Vasseur, L. Dobrzynski, "Two-dimensional phononic crystal with tuneable narrow pass band: Application to a waveguide with selective frequency", J. Appl. Phys. 94 (2003), pp. 13081311.

12 V. Laude, L. Robert, W. Daniau, A. Khelif, S. Ballandras, "Surface acoustic wave trapping in a periodic array of mechanical resonators", Appl. Phys. Lett. 89 (2006), pp. 083515.

13 P. Dainese, P.S.J. Russell, N. Joly, J.C. Knight, G.S. Wiederhecker, H.L. Fragnito, V. Laude, A. Khelif, "Stimulated Brillouin scattering from multi-GHz-guided acoustic phonons in nanostructured photonic crystal fibres", Nat. Phys. 2 (2006), pp. 388-392.

14 R. Lucklum, "Phononic crystal sensor", 2008 IEEE Freq. Contr. Symp., Honolulu, Proc., pp. 85-90.

15 R. Lucklum. I. Hauer, "Transmission Properties of a 1D Resonant Cavity", 2009 EFTF-IEEE IFCS Joint Conference, Besancon, France, Proc., pp 248-253.

16 C. Qiu, Z. Liu, J. Mei, M. Ke, "The layer multiple-scattering method for calculating transmission coefficients of 2D phononic crystals", Solid State Commun. 134 (2005), pp. 765-770.

17 W. Schaaffs: in Landolt-Boernstein NS5, p. 18, Springer, Berlin-Heidelberg-New York, 1967.

18 D. Venkatesulu, P. Venkatesu, M. V. Prabhakara Rao, "Speed of Sound and Isentropic Compressibilities of Trichloroethylene with Branched Alcohols at 303.15 K", J. Chem. Eng. Data 42 (1997), pp. 1145-1146.

19 R.K. Bachu, M.K. Patwari, S. Boodida, S.J. Tangeda, S. Nallani, "Densities, viscosities and speed of sound of binary mixtures of phenylacetonitrile with some aliphatic alcohols at 308.15 K", Indian J. Chem. 47A (2008), pp. 10261031.

20 R. Lucklum, J. Li. "Phononic Crystals for Liquid Sensor Applications", Meas. Sci. Techn. 20 (2009), pp. 124014.

21 M. Zubtsov, R. Lucklum, "Tailoring 2D phononic crystal sensor properties by lattice symmetry reduction", Procedia Engineering 5 (2010), pp. 1284-1287; IEEE Ultrason. Symp., San Diego, Proc., in print.

22 B. Hou, J. Mei, M. Ke, Z. Liu, J. Shi, W. Wen, "Experimental determination for resonance-induced transmission of acoustic waves through sub-wavelength hole arrays", J. Appl. Phys., 104, (2008), pp. 014909.

23 R. Lucklum, J. Li, M. Zubtsov, "1D and 2D Phononic Crystal Sensors", Procedia Engineering 5 (2010), pp. $436-439$. 